\title{
Liraglutide therapy beyond glycemic control: an observational study in Indian patients with type 2 diabetes in real world setting
}

This article was published in the following Dove Press journal:

International Journal of General Medicine

12 April 2012

Number of times this article has been viewed

Jothydev Kesavadev

Arun Shankar

Gopika Krishnan

Sunitha Jothydev

Jothydev's Diabetes Research Center, JDC Junction, Trivandrum, Kerala, India 695032

Video abstract

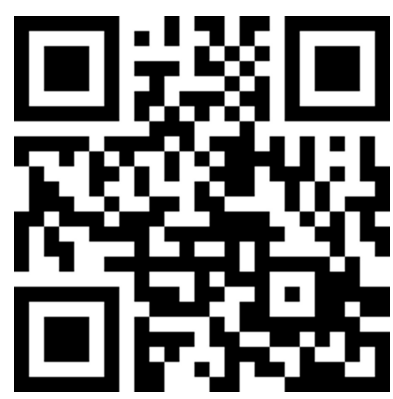

Point your SmartPhone at the code above. If you have a QR code reader the video abstract will appear. Or use: http://dvpr.es/rbstricker

Correspondence: Jothydev Kesavadev Jothydev's Diabetes Research Centre, Trivandrum 695032, Kerala, India

Tel +91 47I 2356200

Fax+9| 47I 2356666

Email jothydev@gmail.com
Background: Liraglutide is an analog of human glucagon-like peptide-1 (GLP-1) and acts as a GLP-1 receptor agonist. Liraglutide is presently used in the treatment of selected patients with type 2 diabetes mellitus (T2DM).

Objective: To assess efficacy and safety of liraglutide in, overweight and obese Indian patients with T2DM.

Methods: A single center, prospective, open-labeled, single-arm, observational study for 24 weeks in a real-world setting. Fourteen overweight and obese patients with T2DM who were clinically suitable for liraglutide therapy received liraglutide injections. The starting dose of liraglutide (Victoza) injection was $0.6 \mathrm{mg} /$ day for 3 days followed by $1.2 \mathrm{mg}$ for next 10 days and finally $1.8 \mathrm{mg}$ /day for 22 weeks. Patients were evaluated at baseline and after 12 and 24 weeks of therapy. Adverse events (AE) noted during course of therapy were recorded. A repeated measure analysis of variance was performed to assess statistical significance.

Results: Fourteen patients were studied for 24 weeks. After 24 weeks of liraglutide therapy, mean fasting and postprandial plasma glucose decreased by $48.5 \mathrm{mg} / \mathrm{dL}$ and $66.71 \mathrm{mg} / \mathrm{dL}$, respectively ( $P=0.002$ and $P=0004$ over 24 weeks, respectively). A mean reduction of $2.26 \%$ of glycosylated hemoglobin was noted $(P<0.001$ over 24 weeks $)$. Mean decrease in body weight of $8.65 \mathrm{~kg}$ and mean decrease in body mass index of $3.26 \mathrm{~kg} / \mathrm{m}^{2}$ was noted $(P<0.001$ over 24 weeks for each parameter). Systolic blood pressure was reduced by $15.15 \mathrm{~mm}$ of $\mathrm{Hg}(P=0.004)$. Significant improvement in total cholesterol, low-density lipoprotein, triglycerides, and serum creatinine was noted. Nine patients reported AEs. The AEs noticed were nausea $(n=6)$, feeling of satiety $(\mathrm{n}=3)$, and vomiting $(\mathrm{n}=1)$. No serious AE or hypoglycemic episodes were observed.

Conclusion: Liraglutide once a day improved overall glycemic control and was well tolerated. Clinically significant reduction in body weight, systolic blood pressure and improvement in lipid profile were noticed with liraglutide therapy in addition to glycemic control.

Keywords: liraglutide, weight loss, type 2 diabetes, obesity, GLP-1 analog, lipid profile, blood pressure, India

\section{Introduction}

A good majority of patients with type 2 diabetes (T2DM) present with concomitant obesity. The increase in prevalence of obesity over the last 20 years closely parallels the increase in prevalence of T2DM. ${ }^{1}$ Cultural changes in terms of food preferences and easy availability of convenience foods, irregular meals, frequent snacking on energydense fast foods, consumption of animal foods, sweetened carbonated drinks, low intake of fiber, monounsaturated fatty acids (MUFAs), and n-3 polyunsaturated fatty acids (PUFAs) are leading to an increased prevalence of obesity in India. ${ }^{2}$ The recently 
published Indian Council of Medical Research - India DIABetes (ICMR-INDIAB) study projected a prevalence rate of 62.4 million and 77.2 million people in India with diabetes and prediabetes, respectively. This study also highlighted the association of abdominal obesity as a predominant risk factor for prediabetes and T2DM. ${ }^{3}$

Weight reduction is the corner stone in the management of T2DM, and is reported to increase insulin sensitivity, reduce blood pressure, improve lipid profile, and reduces markers of inflammation, coagulation, and endothelial dysfunction. ${ }^{4}$ As T2DM is a complex, multifactorial, and progressive disorder, maintaining an individualized glycemic target is always challenging for both patient and the treating physician. Therefore there is a need for newer drugs in the effective management of T2DM. Incretin-based therapies for treatment of T2DM are a recent addition in the therapeutic armamentarium of diabetes management.

Liraglutide (Victoza; Novo Nordisk A/S, Bagsværd, Denmark) is an analog of human glucagon-like peptide-1 (GLP-1) and acts as a GLP-1 receptor agonist. It is indicated as an adjunct to diet and exercise to improve glycemic control in adults with T2DM. ${ }^{5}$ Liraglutide is available in India since July 2010. We present an observational study of liraglutide in newly diagnosed overweight and obese Indian patients with T2DM from a diabetes research center in India.

\section{Materials and methods}

This was a single center, prospective, open-labeled, singlearm, observational study of 24 weeks duration in a real-world setting. T2DM patients with coexisting overweight and obesity clinically suitable for liraglutide therapy were enrolled and managed with Diabetes Tele Management System $\left(\text { DTMS }^{\circledR}\right)^{6}$ at Jothydev's Diabetes Research Center at Trivandrum, Kerala, India. DTMS ${ }^{\circledR}$ is a unique telemedicine tool in vogue since 1998. It consists of special software, networked computers, and a multidisciplinary team that follows up diabetes patients via telephone or Internet and provides continuous education, modification of dosages and lifestyles, and ensures compliance with therapeutic instructions. The team comprises of more than 20 trained doctors, educators, dietitians, nurses, pharmacists, and a psychologist.

Patients with known contraindications for liraglutide therapy (based on prescribing information of Victoza ${ }^{\circledR}$ injection) were excluded from the study. This study complied with the principles of the Declaration of Helsinki. Formal written informed consent for participation in the study was obtained from each patient.
Individualized diet modification and exercise plan was discussed and prescribed for all patients. All patients received liraglutide therapy in addition to their existing antidiabetic therapy. The starting dose of liraglutide (Victoza) injection was $0.6 \mathrm{mg} /$ day for 3 days which was escalated to $1.2 \mathrm{mg}$ for next 10 days and finally $1.8 \mathrm{mg} /$ day for 22 weeks. There was no washout period for the drugs previously used in these patients and the duration of therapy was 24 weeks (6 months).

All patients were evaluated at baseline, after 12 weeks and after 24 weeks of therapy. Physical examination, glycemic control (fasting and postprandial plasma glucose [FPG; PPG], glycosylated hemoglobin $\left.\left[\mathrm{HbA}_{1 \mathrm{c}}\right]\right)$, lipid profile and other relevant investigations as required were repeated.

The doses of other antidiabetic drugs were modified as and when required via DTMS ${ }^{\circledR}$. After 24 weeks of therapy, liraglutide was tapered and stopped and patients were continued on minimal/no drugs and lifestyle modifications.

The safety and tolerability of liraglutide was assessed continuously during the study and any adverse event (AE) noted during course of therapy was recorded. Additionally kidney function was evaluated at every visit.

\section{Statistical methods}

Descriptive statistical analysis has been carried out in the present study. Results on continuous measurements are presented on mean \pm standard deviation (min-max) and results on categorical measurements are presented in percentages. Statistical significance was assessed at a $5 \%$ level. The following assumptions on data were made, Assumptions: (i) dependent variables were normally distributed, (ii) samples drawn from the population were random and samples of the cases were independent.

Table I Baseline demographic data

\begin{tabular}{lll}
\hline Sr no & Parameter & Observed value \\
\hline I. & Number of patients & 14 \\
2. & Male:female & $7: 7$ \\
3. & Age (years, mean $\pm \mathrm{SD})$ & $43.64 \pm 13.89$ \\
4. & Duration of T2DM (weeks, mean $\pm \mathrm{SD})$ & $33.50 \pm 80.28$ \\
5. & Height $(\mathrm{cm}$, mean $\pm \mathrm{SD})$ & $165.36 \pm 14.57$ \\
6. & Weight $(\mathrm{kg}$, mean $\pm \mathrm{SD})$ & $90.7 \mathrm{I} \pm 1 \mathrm{I} .80$ \\
7. & Body mass index $\left(\mathrm{kg} / \mathrm{m}^{2}\right.$, mean $\left.\pm \mathrm{SD}\right)$ & $33.49 \pm 5.15$ \\
8. & Systolic blood pressure & $137.86 \pm 13.89$ \\
& (mmHg, mean $\pm \mathrm{SD})$ & \\
9. & Diastolic blood pressure & $78.29 \pm 8.77$ \\
& $(\mathrm{mmHg}$, mean $\pm \mathrm{SD})$ & \\
\hline
\end{tabular}

Abbreviation: SD, standard deviation. 
Table 2 Improvement in glycemic control

\begin{tabular}{llccc}
\hline & Baseline & After I2 weeks & After 24 weeks & P value (Change) \\
\hline FBG $(\mathrm{mg} / \mathrm{dL})$ & $147.57 \pm 46.68$ & $102.36 \pm 29.90$ & $99.07 \pm 22.55$ & $0.002^{* *}$ \\
(Quadratic change) & & $0.004^{* *}$ \\
PPBG $(\mathrm{mg} / \mathrm{dL})$ & $167.57 \pm 77.44$ & $117.50 \pm 40.79$ & $100.86 \pm 35.72$ & $($ Quadratic change) \\
$\mathrm{HbA}_{\mathrm{Ic}}(\%)$ & $7.78 \pm 1.74$ & $6.42 \pm 1.10$ & $5.52 \pm 0.53$ & $<0.00 I^{* *}$ \\
& & & & (Linear change) \\
\hline
\end{tabular}

Notes: Results are presented in mean \pm SD, $P$ values are repeated measures analyses of variance; **strongly significant.

Abbreviations: FBG, fasting blood glucose; PPBG, postprandial blood glucose; HbA

Repeated measures analysis of variance has been performed to find the significance of change of study variables over baseline, after 12 weeks, and after 24 weeks. The difference was considered as significant with $P$ values of $<0.1$ to 0.05 , moderately significant with $P$ values of $<0.05$ to 0.01 and strongly significant with $P$ value of $<0.01$. $^{7-10}$

\section{Statistical software}

The statistical software, SAS (version 9.2; SAS Institute, Cary, NC) and R environment (version 2.11.1; R Foundation for Statistical Computing, Vienna, Austria), were used for the analysis of the data.

\section{Results}

Fourteen patients (seven male and seven female) receiving liraglutide therapy were followed-up for 24 weeks. Baseline demographic data is shown in Table 1. Mean (SD) age of 43.64 (13.89) years and majority (12/14) of patients had T2DM of less than 12 weeks duration. The mean weight and body mass index (BMI) were $90.71(11.80) \mathrm{kg}$ and 33.49 (5.15) $\mathrm{kg} / \mathrm{m}^{2}$, respectively.

With 12 weeks of liraglutide therapy, mean decrease in FPG and $P B G$ was $45.21 \mathrm{mg} / \mathrm{dL}$ and $50.07 \mathrm{mg} / \mathrm{dL}$, respectively. After 24 weeks of therapy, FPG and PPG decreased by $48.5 \mathrm{mg} / \mathrm{dL}$ and $66.71 \mathrm{mg} / \mathrm{dL}$, respectively (Table 2 and

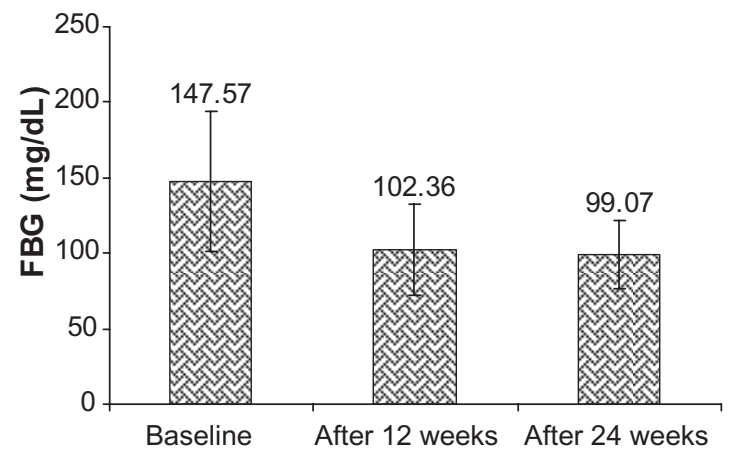

Figure I Reduction in fasting and post-prandial blood glucose.
Figure 1). A quadratic change in reduction of FPG and PPG was noted over 24 weeks $(P=0.002$ and 0.004 , respectively).

Similarly $\mathrm{HbA}_{1 \mathrm{c}}$ decreased by $1.36 \%$ and $2.26 \%$ after 12 and 24 weeks of therapy, respectively. A linear reduction in $\mathrm{HbA}_{\mathrm{lc}}$ over 12 and 24 weeks was noted $(P<0.001)$ (Table 2 and Figure 2).

Significant reduction in body weight and BMI were noted over 24 weeks $\left(-8.65 \mathrm{~kg}, P<0.001\right.$ and $-3.26 \mathrm{~kg} / \mathrm{m}^{2}$, $P<0.001$ ) (Table 3 and Figure 3).

A significant decrease in systolic blood pressure (SBP) of $15.15 \mathrm{~mm}$ of $\mathrm{Hg}(P=0.004)$ and decrease in diastolic blood pressure (DBP) of $1.29 \mathrm{~mm}$ of $\mathrm{Hg}$ was noted over 24 weeks of therapy (Table 4).

Prior to starting liraglutide therapy, subjects were on various therapeutic regimens. With start of liraglutide therapy, concomitant drugs were modified. After 12 and 24 weeks of liraglutide therapy, the requirement of other antidiabetic drugs were modified via $\mathrm{DTMS}^{\circledR}$ as and when required (Table 5). Significant improvement was noted in total cholesterol, high-density lipoprotein, and triglycerides over 24 weeks of therapy (Table 6). Significant reduction in serum creatinine was also noted (Table 6).

Nine patients reported AEs. The most common AE was nausea $(n=6)$ followed by feeling of satiety $(n=3)$,

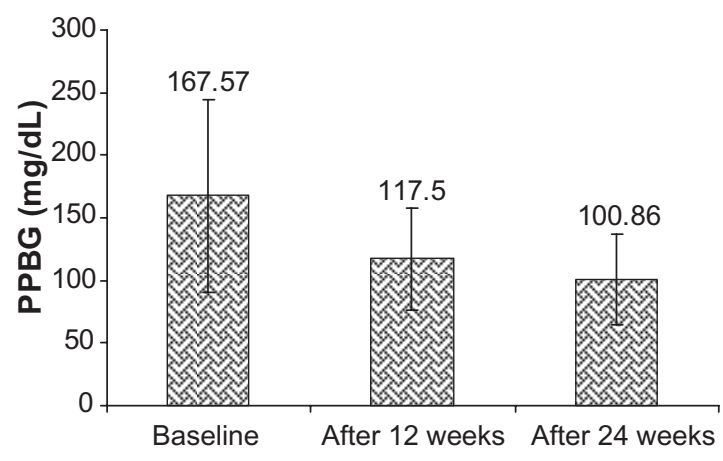




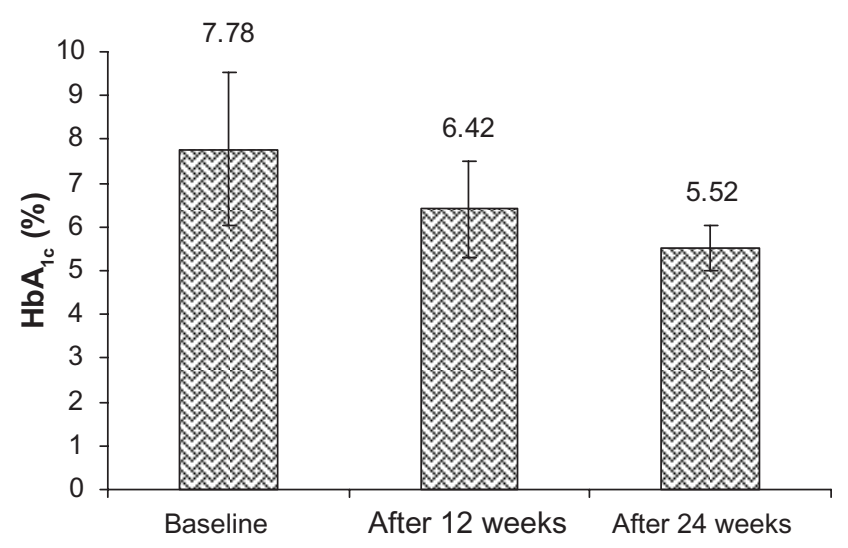

Figure 2 Reduction in $\mathrm{HbA}_{1 \mathrm{c}}$.

and vomiting $(\mathrm{n}=1)$. Nausea persisted for $1-2$ weeks in five of six patients, while in one patient it subsided after 5 days of therapy. Vomiting was seen in one subject for the first 3 days, while feeling of satiety was noticed for 1 month in three patients. All AEs were of minimal clinical significance and self-limiting. None of the patients reported any serious AE.

\section{Discussion}

Currently conventional diabetes management does not address the diminution of the incretin effect, recognized as one of the key pathophysiological defects in T2DM. Since insulin resistance and obesity are key pathophysiological factors resulting in the development of T2DM, therapies addressing these may be of potential importance. Presently, except metformin, treatment with sulphonylureas, thiazolidinediones, and insulin eventually result in weight gain during the course of therapy. GLP-1 analogs are a new group of therapeutic agents which help attain normoglycemia along with weight loss.

Liraglutide (Victoza; Novo Nordisk A/S) is a modified form of human GLP-1 analog acting as a GLP-1 receptor

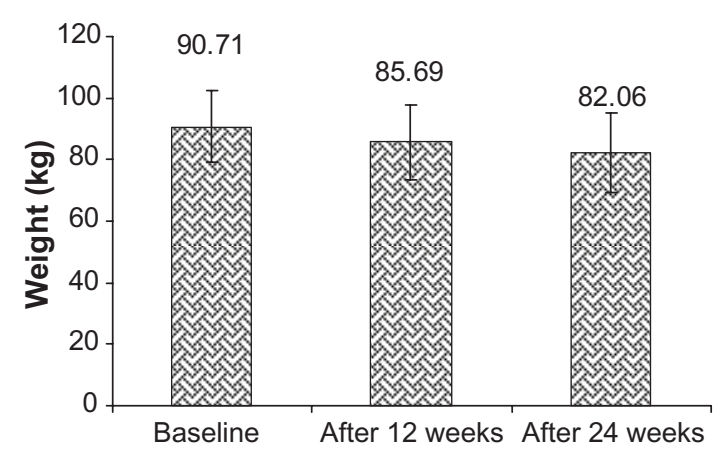

Figure 3 Reduction in weight and body mass index.
Table 3 Decrease in weight and body mass index

\begin{tabular}{lll}
\hline & $\begin{array}{l}\text { Weight } \\
\mathbf{( k g )}\end{array}$ & $\begin{array}{l}\text { Body mass index } \\
\left(\mathbf{k g} / \mathbf{m}^{2}\right)\end{array}$ \\
\hline Baseline & $90.7 \mathrm{I} \pm 11.80$ & $33.49 \pm 5.15$ \\
After I2 weeks of therapy & $85.69 \pm 12.20$ & $31.61 \pm 5.00$ \\
After 24 weeks of therapy & $82.06 \pm 12.86$ & $30.23 \pm 5.01$ \\
$P$ value & $<0.00 I^{* *}$ & $<0.001$ ** \\
\hline
\end{tabular}

Notes: Results are presented in mean \pm SD, $P$ values are repeated measures analyses of variance; **strongly significant.

agonist and is available in India for more than a year. Many studies evaluated and established the efficacy and safety of liraglutide in T2DM. These studies reported that liraglutide decreases $\mathrm{HbA}_{1 \mathrm{c}}$ in monotherapy and in combination with other antidiabetic drugs; inhibits glucagon secretion, decreases gastric emptying, and also results in weight loss. ${ }^{11}$

We evaluated the efficacy and safety of liraglutide in overweight and obese Indian T2DM patients. A majority of the patients $(85.71 \%(12 / 14))$ had been diagnosed with diabetes less than 12 weeks prior to starting liraglutide therapy. A single-center study, small sample size, open label, and noncomparative design are the limitations of the present study and therefore our results must be interpreted with caution. Additionally we could not assess insulin resistance and beta cell function due to nonavailability of equipment and resources. Nevertheless, the results represent data from a real-life clinical setting and appears clinically significant with some interesting findings of the effect of liraglutide therapy in management of overweight and obese T2DM patients.

This limited group of patients reported slightly more decrease in $\mathrm{HbA}_{1 \mathrm{c}}(2.26 \%$ at 24 weeks) compared to other published LEAD studies. ${ }^{12-17}$

The effects of liraglutide on body weight appear quite interesting in this study. After, 12 weeks of liraglutide

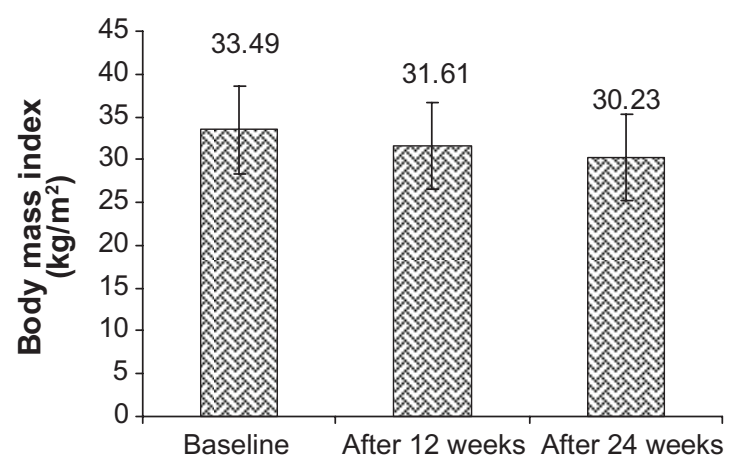


Table 4 Improvement in blood pressure

\begin{tabular}{lll}
\hline & $\begin{array}{l}\text { Systolic } \\
\text { blood pressure } \\
(\mathbf{m m H g})\end{array}$ & $\begin{array}{l}\text { Diastolic } \\
\text { blood pressure } \\
(\mathbf{m m H g})\end{array}$ \\
\hline Baseline & $137.86 \pm 13.89$ & $78.29 \pm 8.77$ \\
After 12 weeks of therapy & $|27.71 \pm 13.8|$ & $76.21 \pm 9.24$ \\
After 24 weeks of therapy & $|22.7| \pm 10.38$ & $77.00 \pm 10.4 \mid$ \\
$P$ value & $0.004 * *$ & 0.767 \\
\hline
\end{tabular}

Notes: Results are presented in mean \pm SD, $P$ values are repeated measures analyses of variance; **strongly significant.

$1.8 \mathrm{mg} /$ day therapy, mean reduction in body weight was $5.02 \mathrm{~kg}$ and after 24 weeks of therapy, total reduction in body weight was $8.65 \mathrm{~kg}(P<0.001)$. Similarly, BMI was decreased by $3.26 \mathrm{~kg} / \mathrm{m}^{2}$ after 24 weeks of therapy $(P<0.001)$. Published evidence from LEAD studies have reported an average decrease of $3.39 \mathrm{~kg}$ weight at 52 weeks of therapy and $3.24 \mathrm{~kg}$ in 26 weeks. ${ }^{14,17}$

Jendle et al reported that there is reduction in fat mass rather than tissue mass with liraglutide therapy. Additionally, it was noted that there was more reduction in visceral fat than subcutaneous abdominal fat. ${ }^{18}$ The weight loss could be as a result of the delayed gastric emptying and early satiety induced by liraglutide. Reduction of weight is a cornerstone in management of T2DM as it is associated with improvement in insulin sensitivity, reduction in blood pressure, and improvement in lipid profile. Therefore a mean reduction of $8.65 \mathrm{~kg}$ body weight over 24 weeks could have significant clinical effects in the management of T2DM.

This study also highlights the place of liraglutide in prediabetes and newly diagnosed patients with T2DM, as the majority of patients (85.71\%) were having diabetes duration of $<12$ weeks. In the present study, glycemic control close to normal levels (mean FPG, PPG, and $\mathrm{HbA}_{1 \mathrm{c}}$ of $99.07 \mathrm{mg} / \mathrm{dL}, 100.86 \mathrm{mg} / \mathrm{dL}$, and $5.52 \%$, respectively) was achieved within 24 weeks of liraglutide therapy. This may be due to the significant reduction in weight along with other effects of liraglutide. This study also points to a possibility of completely reversing T2DM leading to no therapy or minimal
Table 5 Antidiabetic and other drugs coprescribed

\begin{tabular}{lllll}
\hline & $\begin{array}{l}\text { Prior to } \\
\text { study }\end{array}$ & Baseline & $\begin{array}{l}\text { After } \\
\text { I2 weeks }\end{array}$ & $\begin{array}{l}\text { After } \\
\text { 24 weeks }\end{array}$ \\
\hline Metformin, (N) & 07 & 13 & 11 & 09 \\
Sulfonylurea, (N) & 02 & 01 & 00 & 00 \\
DPP-4 I, (N) & 02 & 00 & 00 & 00 \\
Statin, (N) & 05 & 09 & 06 & 04 \\
ARB, (N) & 02 & 03 & 03 & 02 \\
Beta-blocker, (N) & 02 & 02 & 02 & 02
\end{tabular}

Notes: Total patients: 14; patient may be taking more than I drug; N: number of patients.

Abbreviations: DPP-4 I, Dipeptidyl peptidase 4 inhibitor; ARB, angiotensin receptor blocker.

therapy after an initial course of liraglutide for 24 weeks. In this study after 24 weeks of therapy liraglutide was tapered and stopped after achieving euglycemia and patients were continued on lifestyle modifications and minimal drugs. These patients are currently being followed up and assessed to check on the long term outcomes and this data would be published at a later stage.

Another interesting finding was the significant reduction in SBP. Over 24 weeks of liraglutide $1.8 \mathrm{mg}$ /day therapy, SBP was reduced by $15.15 \mathrm{~mm}$ of $\mathrm{Hg}(P=0.004)$. Additionally there was positive improvement in lipid profile in terms of significant reduction in total cholesterol $(P<0.001)$, lowdensity lipoproteins $(P=0.001)$ and serum triglycerides $(P=0.002)$ over 24 weeks. As these are independent risk factors for cardiovascular (CV) disease, reduction in BP and improvement in lipid profile reported by liraglutide therapy may be associated with improved CV outcome in T2DM. Reported evidence suggests that liraglutide must not be administered in moderate to severe renal failure and there have been postmarketing reports of acute renal failure and worsening of chronic renal failure, which may sometimes require hemodialysis in liraglutide-treated patients. However significant reduction in serum creatinine was noted in this study $(P=0.027)$.

We also observed that addition of liraglutide injection reduced the requirement of other antidiabetic drugs.

Table 6 Improvement in lipid profile and kidney function test

\begin{tabular}{|c|c|c|c|c|}
\hline & Baseline & After 12 weeks & After 24 weeks & $P$ value \\
\hline Total cholesterol, (mg/dL) & $166.07 \pm 38.53$ & $134.5 \pm 25.35$ & $125.14 \pm 22.04$ & $<0.001 * *$ \\
\hline $\mathrm{HDL},(\mathrm{mg} / \mathrm{dL})$ & $43.14 \pm 11.98$ & $42.21 \pm 9.82$ & $41.21 \pm 9.48$ & 0.236 \\
\hline LDL, (mg/dL) & $94.21 \pm 30.92$ & $74.71 \pm 25.26$ & $64.5 \pm 21.17$ & $0.00 \mathrm{I} * *$ \\
\hline TG, (mg/dL) & $129.07 \pm 56.34$ & $103.64 \pm 43.37$ & $93.36 \pm 36.68$ & $0.002 * *$ \\
\hline Serum creatinine, $(\mathrm{mg} / \mathrm{dL})$ & $0.81 \pm 0.12$ & $0.78 \pm 0.07$ & $0.75 \pm 0.05$ & $0.027 *$ \\
\hline Urine albumin, (mg/dL) & $15.64 \pm 14.57$ & $10.05 \pm 4.05$ & $8.71 \pm 0.91$ & 0.108 \\
\hline
\end{tabular}

Notes: Results are presented in mean \pm SD, $P$ values are repeated measures analyses of variance; *moderately significant; **strongly significant. Abbreviations: HDL, high-density lipoprotein; LDL, low-density lipoprotein; TG, triglycerides. 
Liraglutide was well tolerated in these Indian patients. There was no episode of hypoglycemia or any other serious AE. All observed AEs were of minor clinical significance and as reported in published literature.

\section{Conclusion}

In this 24-week, prospective, open-labeled, single-center study, liraglutide once a day in combination with metformin over a period of 6 months significantly improved glycemic control to normal level and was well tolerated in the majority of patients. Additionally clinically significant reduction in body weight, systolic blood pressure and improvement in lipid profile were noticed with liraglutide. However these results need to be interpreted with caution and need to be replicated in large multicentric randomized controlled trials for establishing the effects of liraglutide beyond glycemic control. The observation that liraglutide therapy results in probable remission of newly diagnosed T2DM needs to be established in randomized controlled trials.

\section{Disclosure}

The authors report no conflicts of interest in this work.

\section{References}

1. Eckel RH, Kahn SE, Ferrannini E, et al. Obesity and type 2 diabetes: what can be unified and what needs to be individualized? J Clin Endocrinol Metab. 2011;96(6):1654-1663.

2. Misra A, Sharma R, Gulati S, et al; National Dietary Guidelines Consensus Group. Consensus dietary guidelines for healthy living and prevention of obesity, the metabolic syndrome, diabetes, and related disorders in Asian Indians. Diabetes Technol Ther. 2011;13(6):683-694.

3. Anjana RM, Pradeepa R, Deepa M, et al; on behalf of the ICMR-INDIAB Collaborative Study Group. Prevalence of diabetes and prediabetes (impaired fasting glucose and/or impaired glucose tolerance) in urban and rural India: Phase I results of the Indian Council of Medical ResearchINdia DIABetes (ICMR-INDIAB) study. Diabetologia. 2011;54(12): 3022-3027.

4. Mitri J, Hamdy O. Diabetes medications and body weight. Expert Opin Drug Saf. 2009;8(5):573-584.

5. Victoza ${ }^{\circledR}$ (liraglutide [rDNA origin] injection). Prescribing information. Available at: http://www.accessdata.fda.gov/drugsatfda_docs/ label/2011/022341s004 lbl.pdf. Accessed September 1, 2011.
6. Kesavadev J, Shankar A, Shamsudeen J, et al. Telefollow up and SMBG via "DTMS" - A cost effective tool for A1c lowering [abstract] Diabetes. 2010;59(Suppl 1):A546.

7. Rosner B. Fundamentals of Biostatistics. 5th ed. Pacific Grove, CA: Duxbury; 2000:80-240.

8. Riffenburgh RH. Statistics in Medicine. 2nd ed. San Diego, CA: Academic Press; 2005:85-125.

9. Sunder Rao PSS, Richard J. An Introduction to Biostatistics: A manual for students in health sciences. New Delhi, India: Prentice Hall of India; 1996:86-160.

10. Eng J. Sample size estimation: How many individuals should be studied? Radiology. 2003;227:309-313.

11. Madsbad S. Liraglutide Effect and Action in Diabetes (LEAD ${ }^{\mathrm{TM}}$ ) trial. Expert Rev Endocrinol Metabol. 2009;4(2):119-129.

12. Marre M, Shaw J, Brändle M, et al; LEAD-1 SU Study Group. Liraglutide, a once-daily human GLP-1 analogue, added to a sulphonylurea over 26 weeks produces greater improvements in glycaemic and weight control compared with adding rosiglitazone or placebo in subjects with type 2 diabetes (LEAD-1 SU). Diabet Med. 2009;26(3): 268-278.

13. Nauck M, Frid A, Hermansen K, et al; LEAD-2 Study Group. Efficacy and safety comparison of liraglutide, glimepiride, and placebo, all in combination with metformin, in type 2 diabetes: the LEAD (liraglutide effect and action in diabetes)-2 study. Diabetes Care. 2009;32(1): 84-90.

14. Garber A, Henry R, Ratner R, et al; LEAD-3 (Mono) Study Group. Liraglutide versus glimepiride monotherapy for type 2 diabetes (LEAD-3 Mono): a randomised, 52-week, phase III, double-blind, parallel-treatment trial. Lancet. 2009;373(9662):473-481.

15. Zinman B, Gerich J, Buse JB, et al; LEAD-4 Study Investigators. Efficacy and safety of the human glucagon-like peptide-1 analog liraglutide in combination with metformin and thiazolidinedione in patients with type 2 diabetes (LEAD-4 Met+TZD). Diabetes Care. 2009;32(7):1224-1230.

16. Russell-Jones D, Vaag A, Schmitz O, et al; Liraglutide Effect and Action in Diabetes 5 (LEAD-5) met+SU Study Group. Liraglutide vs insulin glargine and placebo in combination with metformin and sulfonylurea therapy in type 2 diabetes mellitus (LEAD-5 met+SU): a randomised controlled trial. Diabetologia. 2009;52(10):2046-2055.

17. Buse JB, Rosenstock J, Sesti G, et al; LEAD-6 Study Group. Liraglutide once a day versus exenatide twice a day for type 2 diabetes: a 26-week randomised, parallel-group, multinational, open-label trial (LEAD-6). Lancet. 2009;374(9683):39-47.

18. Jendle J, Nauck MA, Matthews DR, et al; LEAD-2 and LEAD-3 Study Groups. Weight loss with liraglutide, a once-daily human glucagon-like peptide-1 analogue for type 2 diabetes treatment as monotherapy or added to metformin, is primarily as a result of a reduction in fat tissue. Diabetes Obes Metab. 2009;11(12):1163-1172.
International Journal of General Medicine

\section{Publish your work in this journal}

The International Journal of General Medicine is an international, peer-reviewed open-access journal that focuses on general and internal medicine, pathogenesis, epidemiology, diagnosis, monitoring and treatment protocols. The journal is characterized by the rapid reporting of reviews, original research and clinical studies across all disease areas.

\section{Dovepress}

A key focus is the elucidation of disease processes and management protocols resulting in improved outcomes for the patient.The manuscript management system is completely online and includes a very quick and fair peer-review system. Visit http://www.dovepress.com testimonials.php to read real quotes from published authors. 